\title{
Strong Fuzzy Chromatic Polynomial (SFCP) of Fuzzy Graphs and Some Fuzzy Graph Structures with Applications
}

\author{
Mamo Abebe Ashebo*, Venkata Naga Srinivasa Rao Repalle \\ Department of Mathematics, Wollega University, Nekemte, Ethiopia \\ Email address: \\ mamoabebe37@gmail.com (M. A. Ashebo),rvnrepalle@gmail.com (V. N. S. R. Repalle) \\ ${ }^{*}$ Corresponding author
}

\section{To cite this article:}

Mamo Abebe Ashebo, Venkata Naga Srinivasa Rao Repalle. Strong Fuzzy Chromatic Polynomial (SFCP) of Fuzzy Graphs and Some Fuzzy Graph Structures with Applications. Pure and Applied Mathematics Journal. Vol. 9, No. 1, 2020, pp. 16-25. doi: 10.11648/j.pamj.20200901.13

Received: December 6, 2019; Accepted: December 24, 2019; Published: January 23, 2020

\begin{abstract}
In fuzzy graph theory, strong arcs have separate importance. Assign different colors to the end nodes of strong arcs in the fuzzy graph is strong coloring. Strong coloring plays an important role in solving real-life problems that involve networks. In this work, we introduce the new concept, called strong fuzzy chromatic polynomial (SFCP) of a fuzzy graph based on strong coloring. The SFCP of a fuzzy graph counts the number of $k$-strong colorings of a fuzzy graph with $k$ colors. The existing methods for determining the chromatic polynomial of the crisp graph are used to obtain SFCP of a fuzzy graph. We establish the necessary and sufficient condition for SFCP of a fuzzy graph to be the chromatic polynomial of its underlying crisp graph. Further, we study SFCP of some fuzzy graph structures, namely strong fuzzy graphs, complete fuzzy graphs, fuzzy cycles, and fuzzy trees. Besides, we obtain relations between SFCP and fuzzy chromatic polynomial of strong fuzzy graphs, complete fuzzy graphs, and fuzzy cycles. Finally, we present dual applications of the proposed work in the traffic flow problem. Once SFCP of a fuzzy graph is obtained, the proposed approach is simple enough and shortcut technique to solve strong coloring problems without using coloring algorithms.
\end{abstract}

Keywords: Fuzzy Graph, Strong Coloring, Strong Fuzzy Chromatic Polynomial, Strong Fuzzy Graph, Complete Fuzzy Graph, Fuzzy Cycle, Fuzzy Tree, Traffic Flow Problems

\section{Introduction}

Fuzzy graph theory is a dominant concept for modeling and solving combinatorial optimization problems which come from different fields. On the basis of Zadeh's fuzzy sets [1], the theory of fuzzy graph was narrated by Rosenfeld [2]. The author has studied the fuzzy analogs of several basic graph theoretical concepts. Bhutani [3] introduced the concept of automorphisms of fuzzy graphs and defined a complete fuzzy graph. Mordeson and Nair [4] have established the necessary and sufficient condition for a fuzzy graph which is cycle to be the fuzzy cycle. The fuzzy tree was characterized by Sunitha and Vijayakumar [5] using its unique maximum spanning tree. Strong arcs in a fuzzy graph were introduced by Bhutani and Rosenfeld [6]. The authors have also studied strong arcs in a fuzzy tree. Classifications of arcs in a fuzzy graph are very helpful to understand the entire structure of the fuzzy graph. Based on the strength of an arc, Mathew and Sunitha [7] have classified arcs in the fuzzy graph. In [8], the authors introduced the strongest strong cycle in fuzzy graphs. Lately, many researchers have actively worked on advancing fuzzy graphs [9-13]. Besides, they have studied fuzzy graph structures including strong, complete, regular fuzzy graph structures [14-16]. More recently, various types of fuzzy graphs such as bipolar fuzzy graphs [17], m-polar fuzzy graphs [18] Pythagorean fuzzy graphs [19], Dombi fuzzy graphs [20], Pythagorean dombi fuzzy graphs [21], Picture fuzzy graphs [22] have studied by different scholars. Moreover, several researchers published their work on applications of types of fuzzy graphs [18, 23$26]$ and index of fuzzy graphs [27, 28].

In fuzzy graph theory, coloring plays a paramount role in solving optimization problems. Munoz et al. [29] introduced the idea of coloring fuzzy graphs. After that, Eslahchi and 
Onagh [30] have defined the fuzzy chromatic number of a fuzzy graph. Several authors including Kishore and Sunitha [31] and Samanta et al. [32], Mahapatra et al. [33] have worked on fuzzy coloring. In [34], Kishore and Sunitha have initiated the concept of the strong coloring of fuzzy graphs based on strong arcs. The authors defined the strong chromatic number of a fuzzy graph. Recently, Rosyida et al. [35] introduced a fuzzy chromatic number in the union of fuzzy graphs. Mamo and Srinivasa Rao [36] introduced the concept of the fuzzy chromatic polynomial of a fuzzy graph based on $\alpha$-cuts.

The concept of strong coloring has a significant role in addressing real-life problems that involve networks. The core contributions of this research article are as follows.

i. As far as we know, there exists no research work on SFCP until now. Hence, in this article, we present the definition of SFCP of a fuzzy graph based on strong coloring.

ii. We establish the necessary and sufficient condition for SFCP of a fuzzy graph and chromatic polynomial of its underlying crisp graph is equivalent.

iii. We study the SFCP of some structures of fuzzy graphs such as strong fuzzy graphs, complete fuzzy graphs, fuzzy cycles, and fuzzy trees.

iv. We obtain relations between SFCP and fuzzy chromatic polynomial of strong fuzzy graphs, complete fuzzy graphs, and fuzzy cycles.

$\mathrm{v}$. We present the definition of a strong fuzzy chromatic number of a fuzzy graph in terms of SFCP.

vi. Dual applications of SFCP in traffic flow problems are described in this article. Also, we suggest SFCP approach to solve strong coloring problems.

\section{Preliminaries}

In this section, we reviewed some basic definitions and concepts on fuzzy graphs, strong arcs and strong coloring of fuzzy graphs, which are important for present work. The following basic definitions and related concepts are taken from [3, 7, 34, 36-38].

Definition 1. A fuzzy graph $G=(V, \sigma, \mu)$ is a triple consisting of a nonempty set $V$ together with a pair of functions $\sigma: V \rightarrow[0,1]$ and $\mu: V x V \rightarrow[0,1]$ such that for all $x, y \in V, \mu(x, y) \leq \sigma(x) \wedge \sigma(y)$. Here, the fuzzy set $\sigma$ is called the fuzzy vertex set of $G$ and $\mu$ the fuzzy edge set of $G$.

We consider a fuzzy graph $G$ is simple and undirected. For notational convenience, we use simply $G$ or $G=(\sigma, \mu)$ to represent the fuzzy graph $G=(V, \sigma, \mu)$.

Definition 2. The fuzzy graph $H=(P, \tau, v)$ is called a fuzz subgraph of $G=(V, \sigma, \mu)$ induced by $P$ if $P \subseteq V, \tau(x)=$ $\sigma(x)$ for all $x \in P$ and $v(x, y)=\mu(x, y)$ for all $x, y \in P$.

Definition 3. We denote the underlying crisp graph of a fuzzy graph $G$ by $G^{*}=\left(\sigma^{*}, \mu^{*}\right)$ where $\sigma^{*}=\{u \in V: \sigma(u)>$ $0\}$ and $\mu^{*}=\{(u, v) \in V x V: \mu(u, v)>0\}$.

Definition 4. The level set of fuzzy set $\sigma$ is defined as $L_{\sigma}=\left\{\alpha: \sigma(u)=\alpha\right.$ for some $\left.u \in \sigma^{*}\right\}$ and the level set of $\mu$ is defined as $L_{\mu}=\left\{\alpha: \mu(u, v)=\alpha\right.$ for some $\left.(u, v) \in \mu^{*}\right\}$. The fundamental set (or level set) of the fuzzy graph
$G=(V, \sigma, \mu)$ is defined as $L=L_{\sigma} \cup L_{\mu}$.

Definition 5. For each $\alpha \in I=L \cup\{0\}, G_{\alpha}$ denotes the $\alpha$ cut of the fuzzy graph $G$ which is the crisp graph $G_{\alpha}=$ $\left(\sigma_{\alpha}, \mu_{\alpha}\right)$ where $\sigma_{\alpha}=\{u \in V / \sigma(u) \geq \alpha\}$ and $\mu_{\alpha}=\{(u, v) \in$ $V \times V / \mu(u, v) \geq \alpha\}$.

Definition 6. A fuzzy graph $G$ is said to be strong fuzzy graph if $\mu(u, v)=\sigma(u) \wedge \sigma(v)$ for all $(u, v)$ in $\mu^{*}$.

Definition 7. A complete fuzzy graph is a fuzzy graph $G=(\sigma, \mu)$ such that $\mu(u, v)=\sigma(u) \wedge \sigma(v)$ for all $u, v \in V$.

Definition 8. Let $G=(\sigma, \mu)$ be a fuzzy graph. Then

(i) $G$ is called a cycle if $G^{*}=\left(\sigma^{*}, \mu^{*}\right)$ is a cycle.

(ii) $G$ is called a fuzzy cycle if $G^{*}=\left(\sigma^{*}, \mu^{*}\right)$ is a cycle and $\nexists$ unique $(u, v) \in \mu^{*}$ such that

$$
\mu(u, v)=\wedge\left\{\mu(u, v) \mid(u, v) \in \mu^{*}\right\}
$$

Definition 9. Let $G=(\sigma, \mu)$ be a fuzzy graph. Then

(i) $G$ is called a tree if $G^{*}=\left(\sigma^{*}, \mu^{*}\right)$ is a tree.

(ii) $G$ is called a fuzzy tree if $G$ has a fuzzy spanning subgraph $F=(\sigma, v)$, which is a tree, such that for all $(u, v) \in \mu^{*} \backslash v^{*}, \mu(u, v)<v^{\infty}(u, v)$. That is, there exists a path in $(\sigma, v)$ between $u$ and $v$ whose strength is greater than $\mu(u, v)$.

Definition 10. A path $P$ in a fuzzy graph $G=(\sigma, \mu)$ is a sequence of distinct vertices $u_{0}, u_{1}, \ldots, u_{n}$ (except possibly $u_{0}$ and $\left.u_{n}\right)$ such that $\mu\left(u_{i-1}, u_{i}\right)>0, i=1,2, \ldots, n$. Here $n$ is called the length of the path.

Definition 11. If $u_{0}=u_{n}$, and $n \geq 3$ then $P$ is called a cycle and $P$ is called a fuzzy cycle, if it contains more than one weakest arc.

Definition 12. The strength of $P$ is defined to be $\bigwedge_{i=1}^{n} \mu\left(u_{i-1}, u_{i}\right)$. In words, the strength of a path is defined as the degree of membership of the weakest arc in $P$. We denote the strength of a path $P$ by $s(P)$.

Definition 13. The strength of connectedness between two vertices $u$ and $v$ is defined as the maximum of the strengths of all paths between $u$ and $v$ and is denoted by $\operatorname{CONN}_{G}(u, v)$ or $\mu^{\infty}(u, v)$. The strongest path joining any two vertices $u, v$ has strength $\mu^{\infty}(u, v)$.

Definition 14. A fuzzy graph $G=(\sigma, \mu)$ is connected if for every $u, v$ in $\sigma^{*}, \mu^{\infty}(u, v)>0$.

Definition 15. An arc $(u, v)$ in $G$ is called $\alpha$-strong if $\mu(u, v)>\operatorname{CONN}_{G-(u, v)}(u, v)$.

Definition 16. An arc $(u, v)$ in $G$ is called $\beta$-strong if $\mu(u, v)=\operatorname{CONN}_{G-(u, v)}(u, v)$.

Definition 17. An arc $(u, v)$ in $G$ is called $\delta$-arc if $\mu(u, v)<\operatorname{CONN}_{G-(u, v)}(u, v)$.

Here, $\operatorname{CONN}_{G-(u, v)}(u, v)$ is the strength of connectedness between $u$ and $v$ in a fuzzy graph obtained from $G$ by deleting the arc $(u, v)$.

Here, a strong arc is either $\alpha$-strong or $\beta$-strong. Also, a $\delta$ arc $(u, v)$ is called a $\delta^{*}$-arc if $\mu(u, v)>\mu(x, y)$ where $\mu(x, y)$ is the weakest arc of $G$.

Definition 18. Consider a fuzzy graph $G=(V, \sigma, \mu)$. The coloring $C: V(G) \rightarrow \mathbb{N}$ (where $\mathbb{N}$ is the set of all positive integers) such that $C(u) \neq C(v)$ if $(u, v)$ is a strong arc $(\alpha$ strong and $\beta$-strong) in $G$ is called strong coloring.

Definition 19. A fuzzy graph $G$ is $k$-strong colorable if 
there exists a strong coloring of $G$ from a set of $k$ colors.

Definition 20. The minimum number $k$ for which $G$ is $k$ strong colorable is called the strong chromatic number of $G$ denoted by $\chi_{s}(G)$.

Definition 21. For a fuzzy graph $G$, the fuzzy chromatic polynomial of $G$ is denoted by $P_{\alpha}^{f}(G, k)$ and defined as the chromatic polynomial of its crisp graphs $G_{\alpha}$, for $\alpha \in I$.

That is, $P_{\alpha}^{f}(G, k)=P\left(G_{\alpha}, k\right), \forall \alpha \in I$.

\section{Strong Fuzzy Chromatic Polynomial of a Fuzzy Graph}

In the sense of arcs, chromatic polynomials of crisp graphs are always strong chromatic polynomials. Since in crisp graph theory, all the arcs are strong by nature. But, in fuzzy graph theory, arcs are different and have separate significance. In this section, we introduce the new concept, strong chromatic polynomial in a fuzzy graph, called strong fuzzy chromatic polynomial (SFCP). Also, we define a strong fuzzy chromatic polynomial of a fuzzy graph based on strong coloring.

A strong fuzzy chromatic polynomial counts the number of strong coloring on the vertices of a fuzzy graph and is defined as follows.

Definition 22. Let $G$ be a fuzzy graph with a positive integer $k$, the number of distinct $k$-strong colorings of $G$ is called strong fuzzy chromatic polynomial (SFCP) of $G$. It is denoted by $P_{s}^{f}(G, k)$.

Example 1. Consider the fuzzy graph $G$ given in Figure 1.

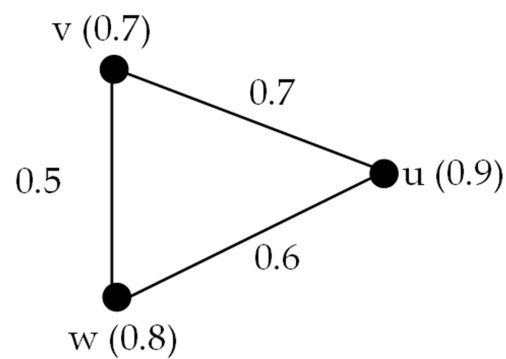

Figure 1. A fuzzy graph $G$.

Here, $\mu(u, v)=0.7, \mu(u, w)=0.6$ and $\mu(v, w)=0.5$. Also, $\operatorname{CONN}_{G-(u, v)}(u, v)=0.5, \operatorname{CONN}_{G-(u, w)}(u, w)=0.5$ and $\operatorname{CONN}_{G-(v, w)}(v, w)=0.6$.

In Figure 1, we found that arc $(u, v)$ is $\alpha$-strong since

$$
(u, v)=0.7>\operatorname{CONN}_{G-(u, v)}(u, v)=0.5
$$

Similarly, arc $(u, w)$ is $\alpha$-strong since

$$
(u, w)=0.6>\operatorname{CONN}_{G-(u, w)}(u, w)=0.5
$$

But $\operatorname{arc}(v, w)$ is $\delta$-arc since

$$
(v, w)=0.5<\operatorname{CONN}_{G-(v, w)}(v, w)=0.6
$$

Here, strong coloring gives $k$ color for $u$ and $k-1$ color for both $v$ and $w$ (See Figure 2).

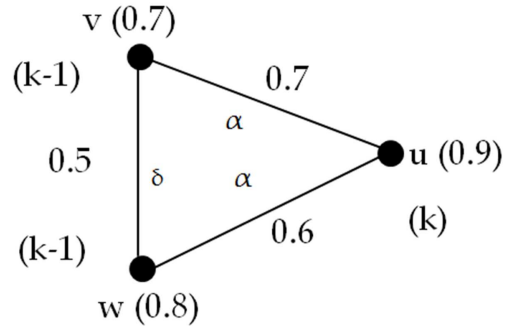

Figure 2. Calculating strong fuzzy chromatic polynomial of $G$.

Hence, the SFCP of $G$ is $k(k-1)(k-1)$. That is, $P_{s}^{f}(G, k)=k(k-1)^{2}$.

Theorem 1 gives the necessary and sufficient condition for SFCP of $G$ and chromatic polynomial of $G^{*}$ are equivalent.

Theorem 1. Let $G$ be a fuzzy graph. Then $P_{s}^{f}(G, k)=$ $P\left(G^{*}, k\right)$ if and only if all the arcs in $G$ are strong.

Proof. Let $G=(\sigma, \mu)$ be a fuzzy graph and $G^{*}=\left(\sigma^{*}, \mu^{*}\right)$ be its underlying crisp graph. Let us first prove if $P_{s}^{f}(G, k)=$ $P\left(G^{*}, k\right)$ then all the arcs in $G$ are strong by contrapositive, suppose that some of the arcs in $G$ are not strong. This shows that there exists at least one $\operatorname{arc}(u, v)$ in $\mu^{*}$ such that $\mu(u, v) \neq \operatorname{CONN}_{G}(u, v)$. Then by Proposition 2 of [6], the arc $(u, v)$ is not strong. This implies that strong coloring gives the same color to the end nodes of the $\operatorname{arc}(u, v)$ in $G$. Thus,

$$
P_{s}^{f}(G, k) \neq P\left(G^{*}, k\right)
$$

Conversely, suppose all the arcs in $G$ are strong. Since all the arcs are strong, then by Proposition 2 of [6], we have $\mu(u, v)=\operatorname{CONN}_{G}(u, v)$ for all arcs $(u, v)$ in $\mu^{*}$ and strong coloring gives different colors to the end vertices of all arcs in $G$ which is analogous to the coloring of the underlying crisp graph $G^{*}$. Hence,

$$
P_{s}^{f}(G, k)=P\left(G^{*}, k\right)
$$

Theorem 2 shows us how to determine the strong fuzzy chromatic polynomial of a fuzzy graph in which some of the arcs are not strong.

Theorem 2. Let $G$ be a fuzzy graph in which some of its arcs are not strong. Then $P_{s}^{f}(G, k)=P\left(H^{*}, k\right)$, where $H$ be a fuzzy subgraph obtained from $G$ by deleting $\delta$-arcs and $H^{*}$ be its underlying crisp graph.

Proof. Let $G$ be a fuzzy graph. Suppose $H$ is a fuzzy subgraph obtained from $G$ by deleting $\delta$-narcs.

Thus, $H$ contains only strong arcs. Therefore, by Theorem 1 we have,

$$
P_{s}^{f}(H, k)=P\left(H^{*}, k\right)
$$

Since $H$ is a fuzzy subgraph of $G$, all the arcs of $H$ are the only strong arcs of $G$. This implies that

$$
P_{s}^{f}(G, k)=P_{s}^{f}(H, k)
$$

Therefore, from equation (1) and (2), we get,

$$
P_{s}^{f}(G, k)=P\left(H^{*}, k\right)
$$




\section{Strong Fuzzy Chromatic Polynomial of Some Fuzzy Graph Structures}

In the section, we study the SFCP of some fuzzy graph structures and their relations with the fuzzy chromatic polynomial.

\subsection{Strong Fuzzy Graphs}

In this subsection, we study the SFCP of strong fuzzy graphs and the relation between SFCP and fuzzy chromatic polynomial of a strong fuzzy graph.

Theorem 3. Let $G$ is a strong fuzzy graph and $G^{*}$ be its underlying crisp graph. Then $P_{s}^{f}(G, k)=P\left(G^{*}, k\right)$.

Proof. Suppose $G$ is a strong fuzzy graph and $G^{*}$ is its underlying crisp graph. Since $G$ is a strong fuzzy graph, then by Theorem 4.1 of [36], all the arcs in $G$ are strong. Then, by Theorem 1, the result holds.

Remark 1. The Converse of Theorem 3 does not hold generally.

Example 2. Let us consider the fuzzy graph $G$, shown in Figure 3.

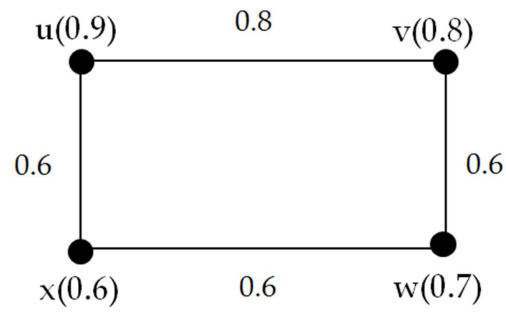

Figure 3. A fuzzy graph $G$.

In $G$, the arc $(u, v)$ is $\alpha$-strong and all the remaining arcs are $\beta$-strong. So, all the arcs in $G$ are strong. Thus, by Theorem 1 we have $P_{s}^{f}(G, k)=P\left(G^{*}, k\right)$. But $G$ is not strong fuzzy graph since $\mu(v, w) \neq \min \{\sigma(v), \sigma(w)\}$, for $(v, w) \in$ $\mu^{*}$.

Remark 2. If all the arcs in a fuzzy graph $G$ are strong, then $G$ need not be a strong fuzzy graph (See Figure 3).

The relation between SFCP and fuzzy chromatic polynomial of a strong fuzzy graph is established below.

Theorem 4. Let $\mathrm{G}$ be a strong fuzzy graph, then $\exists \alpha \in L$ such that $P_{s}^{f}(G, k)=P_{\alpha}^{f}(G, k)$.

Proof. Let $G$ be a strong fuzzy graph. Let $G^{*}$ be the underlying crisp graph of $G$. Let $L$ be a level set of $G$. If we take $\alpha \in L$ and $\alpha=\min (L)$ and since $G$ is a fuzzy graph.

Then by Theorem 40 of [36],

$$
P_{\alpha}^{f}(G, k)=P\left(G^{*}, k\right)
$$

Since $G$ is a strong fuzzy graph, then by Theorem 2, we have

$$
P_{s}^{f}(G, k)=P\left(G^{*}, k\right)
$$

Therefore, from equation (3) and (4), the result holds immediately.

\subsection{Complete Fuzzy Graphs}

In this subsection, the SFCP of complete fuzzy graphs and the relation between SFCP and fuzzy chromatic polynomial of a complete fuzzy graph are studied.

Theorem 5. Let $G$ be a complete fuzzy graph with $n$ vertices. Then $P_{s}^{f}(G, k)=P\left(K_{n}, k\right)$, where $K_{n}$ is a complete crisp graph with $n$ vertices.

Proof. Suppose $G$ is a complete fuzzy graph with n vertices. Then all arcs of a complete fuzzy graph are strong by Proposition 3.13 of [3] and Proposition 2 of [6]. Then, by Theorem 1 we have $P_{s}^{f}(G, k)=P\left(G^{*}, k\right)$ and by Lemma 44 of [36], the result holds immediately.

Remark 3. The Converse of Theorem 5 need not be true. The equation $P_{s}^{f}(G, k)=P\left(K_{n}, k\right)$ does not imply that $G$ is a complete fuzzy graph.

Example 3. Consider the fuzzy graph $G$ shown in Figure 4.

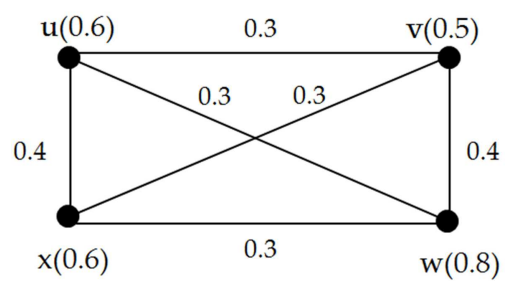

Figure 4. A fuzzy graph $G$.

In $G$, the $\operatorname{arcs}(u, x)$ and $(v, w)$ are $\alpha$ - strong and the remaining arcs are $\beta$-strong. So, all the arcs in $G$ are strong. Therefore, by Theorem 1, we have

$$
P_{s}^{f}(G, k)=P\left(G^{*}, k\right)
$$

which is equal to $P\left(K_{4}, k\right)$. But, $G$ is not a complete fuzzy graph.

Remark 4. If all the arcs in a fuzzy graph $G$ are strong, $G$ is not necessarily a complete fuzzy graph. (See Figure 4)

Remark 5. A complete fuzzy graph is strong whereas a strong fuzzy graph need not be complete.

Remark 6. If a fuzzy graph $\mathrm{G}$ is strong or/and complete fuzzy graph, then $P_{s}^{f}(G, k)=P\left(G^{*}, k\right)$.

The relation between SFCP and fuzzy chromatic polynomial of a complete fuzzy graph is established in Theorem 6.

Theorem 6. If $G$ is a complete fuzzy graph, then there exist $\alpha \in I$ such that $P_{s}^{f}(G, k)=P_{\alpha}^{f}(G, k)$.

Proof. Suppose $G$ is a complete fuzzy graph with n vertices. Define $I=L \cup\{0\}$, where $L$ is the level set of $G$. If $\alpha=0$ and since $G$ is a fuzzy graph, then by Theorem 39 of [36].

$$
P_{\alpha}^{f}(G, k)=P\left(K_{n}, k\right)
$$

Since $G$ is a complete fuzzy graph, by Theorem 5 we have

$$
P_{s}^{f}(G, k)=P\left(K_{n}, k\right)
$$

Therefore, from equation (6) and (7), we get,

$$
P_{s}^{f}(G, k)=P_{\alpha}^{f}(G, k)
$$


Similarly, the result holds for $\alpha=\min (L)$. Hence, for a complete fuzzy graph $G$, there are $\alpha \in I$ such that $P_{s}^{f}(G, k)=P_{\alpha}^{f}(G, k)$.

\subsection{Fuzzy Cycles}

In this subsection, the SFCP of a fuzzy cycle and the relation between SFCP and fuzzy chromatic polynomial of a fuzzy cycle are discussed.

In fuzzy cycle $G$, there are no $\delta$-arcs. In other words, the weakest arcs in $\mathrm{G}$ are $\beta$-strong and all the remaining arcs are $\alpha$-strong [6].

Theorem 7. Let $G$ be a fuzzy graph such that $G^{*}$ is a cycle. Then $G$ is a fuzzy cycle if and only if $P_{s}^{f}(G, k)=P\left(G^{*}, k\right)$.

Proof. Let $G$ be a fuzzy graph such that $G^{*}$ is a cycle. Suppose $G$ is a fuzzy cycle. Since $G$ is a fuzzy cycle, then $G$ contains only strong arcs [7]. Therefore, by Theorem 1, the result immediately holds.

On the other hand, we shall prove by contrapositive of if $P_{s}^{f}(G, k)=P\left(G^{*}, k\right)$ then $G$ is a fuzzy cycle. We want to show $P_{s}^{f}(G, k) \neq P\left(G^{*}, k\right)$ whenever $G$ is not fuzzy cycle. Since $G$ is not a fuzzy cycle, there is at least one $\operatorname{arc}(u, v)$ in $G$ such that $\mu(u, v) \neq \mu^{\infty}(u, v)$. This implies that arc $(u, v)$ is not strong. Therefore, strong coloring assigns the same color to the end nodes of arc $(u, v)$. This implies that $P_{s}^{f}(G, k) \neq P\left(G^{*}, k\right)$. Therefore, if $P_{s}^{f}(G, k)=P\left(G^{*}, k\right)$ then $G$ is a fuzzy cycle.

Here, Theorem 7 gives the necessary and sufficient condition for SFCP of fuzzy graph $G$ such that $G^{*}$ is a cycle to be the chromatic polynomial of $G^{*}$.

Remark 7. Let $G$ be a fuzzy graph with $n$ vertices such that $G^{*}$ is a cycle. Then $G^{*}=C_{n}$, where $C_{n}$ is a cycle crisp graph with $n$ vertices.

Corollary 1 . Let $G$ be a fuzzy graph with $n$ vertices such that $G^{*}$ is a cycle. If $G$ is a fuzzy cycle, then $P_{s}^{f}(G, k)=$ $P\left(C_{n}, k\right)$.

Proof. The result immediately holds from Theorem 7 and Remark 52 of [36].

The relation between SFCP and fuzzy chromatic polynomial of a fuzzy cycle is established below.

Theorem 8 . Let $G$ be a fuzzy graph and $G^{*}$ be a cycle. If $G$ is a fuzzy cycle, then there exist $\alpha \in L$ such that $P_{s}^{f}(G, k)=$ $P_{\alpha}^{f}(G, k)$.

Proof. Let $G$ be a fuzzy graph such that $G^{*}$ is a cycle. Suppose $G$ is a fuzzy cycle. Since $G$ is a fuzzy cycle. Then by Theorem 7, we get

$$
P_{S}^{f}(G, k)=P\left(G^{*}, k\right)
$$

Now take $\alpha=\min (L)$, where $\mathrm{L}$ is the fundamental set of $G$, by Theorem 40 of [36], we have

$$
P_{\alpha}^{f}(G, k)=P\left(G^{*}, k\right)
$$

From equations (8) and (9), we get $P_{s}^{f}(G, k)=P_{\alpha}^{f}(G, k)$. Therefore, for a fuzzy cycle $G$, there exists $\alpha \in L$ such that SFCP of a fuzzy cycle is equal to the fuzzy chromatic polynomial of a fuzzy cycle.

\subsection{Fuzzy Trees}

In this subsection, we discuss the SFCP of a fuzzy tree and a fuzzy graph $G$ whose $G^{*}$ is a tree and not the tree.

Theorem 9. Let $G$ be a fuzzy graph such that $G^{*}$ is a tree. Then $P_{s}^{f}(G, k)=P\left(G^{*}, k\right)$.

Proof. Let $G$ be a fuzzy graph such that $G^{*}$ is a tree. $G$ is a tree by Definition 9 . Since $G^{*}$ is a tree, clearly, all the arcs of $G$ are fuzzy bridges. Then all the arcs in $G$ are strong by Proposition 2 of [6] and Theorem 4 of [5]. Therefore, the result holds by Theorem 1 .

Remark 7. For fuzzy graph $G$ with n vertices such that $G^{*}$ is a tree, then $G^{*}=T_{n}$, where $T_{n}$ is a tree with $\mathrm{n}$ vertices. Moreover, by Theorem 9 , we have $P_{s}^{f}(G, k)=P\left(T_{n}, k\right)$.

Theorem 10 gives the SFCP of a fuzzy tree is equal to the SFCP of its maximum spanning tree.

Theorem 10. Let $G$ be a fuzzy graph such that $G^{*}$ is not a tree. If $G$ is a fuzzy tree, then $P_{s}^{f}(G, k)=P_{s}^{f}(T, k)$, where $T$ is the maximum spanning the tree of $G$.

Proof. Let $G$ be a fuzzy graph and $G^{*}$ be not a tree. Suppose $G$ is a fuzzy tree. Since $G$ is a fuzzy tree, then $G$ has a unique maximum spanning tree $\mathrm{T}$ by Theorem 10 of [5].

Since $G$ is a fuzzy tree and $G^{*}$ is not a tree, by Theorem 5 of [5] $G$ consists of at least one $\delta$-arc. Then, by Proposition 4 of [6], the strong arcs of $G$ are $\operatorname{arcs}$ of $T$ of $G$. This implies that all the arcs of $T$ are strong.

Hence, the strong coloring of $G$ is exactly the same as the strong coloring of $T$ of $G$. Hence, $P_{s}^{f}(G, k)=P_{s}^{f}(T, k)$.

Example 4. Consider the following fuzzy tree $G$ in Figure 5 and its maximum spanning tree $T$ in Figure 6.

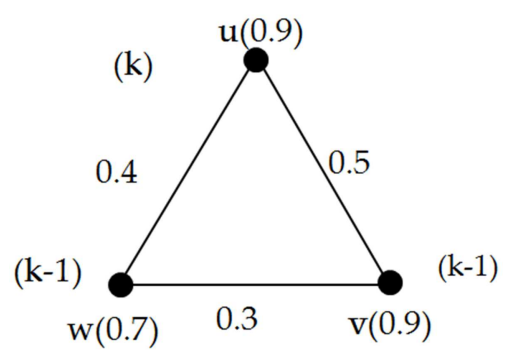

Figure 5. A fuzzy tree $G$.

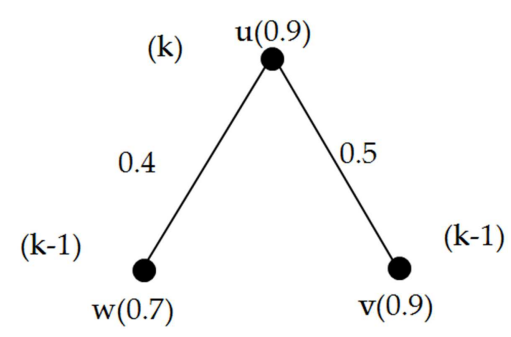

Figure 6. A maximum spanning tree $T$ of $G$.

In Figure 5, arc $(u, v)$ and $\operatorname{arc}(u, w)$ are $\alpha$-strong but $(v, w)$ is $\delta$-arc, whereas, in Figure 6 , both $\operatorname{arcs}(u, v)$ and $(u, w)$ are $\alpha$-strong which are strong arcs of a fuzzy tree $G$ in Figure 5. In $G$ and $T$, vertex $u$ can be strong colorable in $k$ ways and vertices $v$ and $w$ can be strong colorable in $k-1$ ways.

Therefore, 


$$
P_{S}^{f}(G, k)=k(k-1)(k-1)=P_{S}^{f}(T, k)
$$

Theorem 11. Let $G$ be a fuzzy tree and $T$ be its maximum spanning tree. Then $P_{s}^{f}(G, k)=P\left(T^{*}, k\right)$, where $T^{*}$ is the underlying crisp graph of $T$.

Proof. Suppose $G$ is a fuzzy tree and $T$ is its maximum spanning tree. Since $G$ is a fuzzy tree, then all the $\operatorname{arcs} \operatorname{in} T$ are strong by Proposition 4 of [6]. Then by Theorem 1, we have

$$
P_{S}^{f}(T, k)=P\left(T^{*}, k\right)
$$

Where $T^{*}$ is the underlying crisp graph of T.

Since $G$ is a fuzzy tree, then by Theorem 10 , we have

$$
P_{s}^{f}(G, k)=P_{s}^{f}(T, k)
$$

Hence, from equation (10) and (11), we get

$$
P_{s}^{f}(G, k)=P\left(T^{*}, k\right)
$$

Remark 8 . Let $G$ be a fuzzy tree. Then $\nexists \alpha \in I$ such that $P_{s}^{f}(G, k)=P_{\alpha}^{f}(G, k)$.

Example 5. Consider the fuzzy tree $G$ in Figure 7 (a) and its maximum spanning tree $T$ of $G$ in Figure 7 (b).

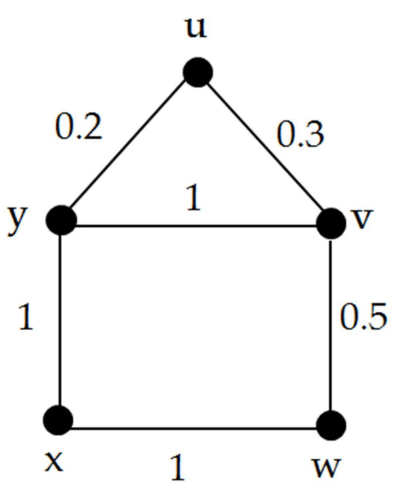

(a)

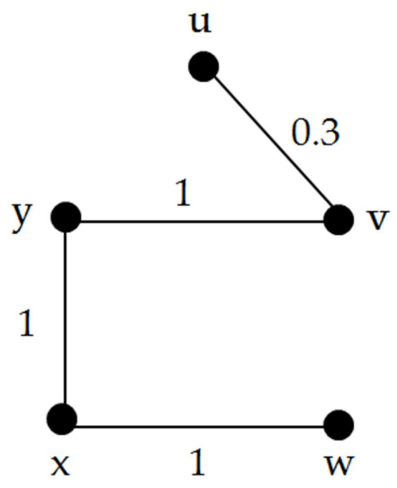

(b)

Figure 7. (a) A fuzzy tree $G$ (b). A maximum spanning tree $T$ of $G$.

By Theorem 10,

$$
P_{s}^{f}(G, k)=P_{s}^{f}(T, k)=k(k-1)^{4} \neq P_{\alpha}^{f}(G, k)
$$

for all $\alpha \in I$.
Theorem 12 . Let $G$ be a fuzzy graph such that $G^{*}$ is a cycle. If $G$ is not fuzzy tree then

$$
P_{S}^{f}(G, k)=P\left(G^{*}, k\right)
$$

Proof. Let $G$ be a fuzzy graph and $G^{*}$ be a cycle. Suppose $G$ is not a fuzzy tree. Then, by Theorem 1 of [4]. $G$ is a fuzzy cycle. Since $G$ is a fuzzy cycle, then the result immediately holds by Theorem 7 .

Besides counting the strong colorings on fuzzy graphs, SFCP can be used to get the strong fuzzy chromatic number of a fuzzy graph.

The following definition tells us how to use the SFCP to find the least number of colors for which a fuzzy graph to be strong colorable.

Definition 23. Let $G$ be a fuzzy graph. The number $k$ is called the strong fuzzy chromatic number of $G, \chi_{s}^{f}(G)$ if there exist the smallest positive integer $k$ such that $P_{s}^{f}(G, k) \neq 0$.

i.e. $k=\chi_{s}^{f}(G)$.

Note that strong fuzzy chromatic polynomials are powerful mathematical tools. Once SFCP of a fuzzy graph is determined, it is a simple and shortcut method for solving strong coloring problems that are modeled by fuzzy graphs.

\section{Applications of Strong Fuzzy Chromatic Polynomial}

In this section, we discuss two applications of the strong fuzzy chromatic polynomial in vehicular traffic flow problems at road intersections. In the first application, we apply our proposed SFCP to find a minimum number of traffic light phases, in the second application to compute the possible number of traffic light patterns with the optimized phases.

\subsection{Application for Finding the Minimum Number of Traffic Light Phases}

In traffic flow problems, phases collect non-conflict movements of traffic flows at road intersections so that the traffic flows being safe and smooth. Phase design is the first step in the traffic signal designing procedure. This shows that phase designing is a very important step since the further steps are affected by it. Besides, a minimum number of phases are very helpful in reducing the waiting time of vehicles and fuel consumption. In this subsection, we apply the proposed SFCP to find the minimum number of traffic light phases at a road intersection.

Now, we demonstrate the first application of SFCP theory to traffic flow problems with the help of Example 6.

Example 6. Consider the traffic flow problem of a city in Figure 8. In this problem, we consider a four-legged intersection through four traffic flows. Each flow can move straight, turn right and turn left at the road intersection. We assume that the number of vehicles at each lane is not equal. Suppose that the traffic volumes of flow 1, flow 2, flow 3 and 
flow 4 are High (0.9), Low (0.2), Medium (0.5) and Low $(0.2)$, respectively.

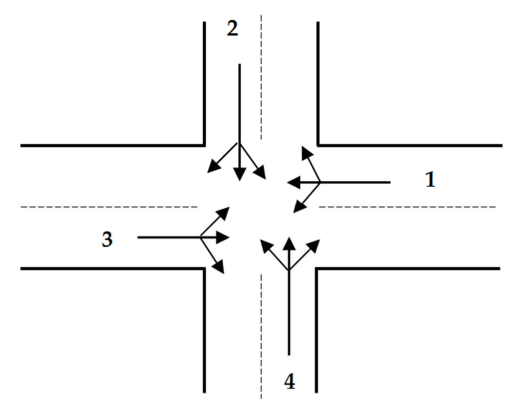

Figure 8. A traffic flow problem of a city at an intersection.

For ensuring safe crossing at road intersections, conflict movements can be managed by using traffic control phases. Now to apply the proposed SFCP method for finding the optimized number of phases, first, we represent the traffic flows (flows 1, 2, $3 \& 4$ ) in Figure 8 by nodes on the graph. Next, connect the pair of flows by an arc if there is a conflicting movement between them. Since the traffic volume of each flow at the roads is not equal and the probability of occurrence of an accident between a pair of conflict flows are also different. To handle these uncertainties and fuzziness which occurred in the problem, we model the traffic flow problem by a fuzzy graph $G=(V, \sigma, \mu)$, where

1. $V=\{1,2,3,4\}$ is fuzzy vertex set,

2. $\sigma: V \rightarrow[0,1]$ is the membership values of the nodes in $V$ and it is characterized by the volume of traffic flows,

3. i.e. $\sigma(1)=0.9, \sigma(2)=0.2, \sigma(3)=0.5 \& \sigma(4)=0.2$,

4. $\mu(i, j)$ is the membership values of the $\operatorname{arcs}$ in $\mu^{*}$ and denote the probability of occurrence of accidents when flows $i$ and $j$ are moving simultaneously at the intersection.

Depending on the possibility of an accident, we consider two cases to model the problem.

Case 1: When $\mu(i, j)=\min \{\sigma(i), \sigma(j)\}$ for $(i, j) \in \mu^{*}$

The fuzzy graph model $G$ for the traffic flow problem in Figure 8 base on Case 1 is shown in Figure 9.

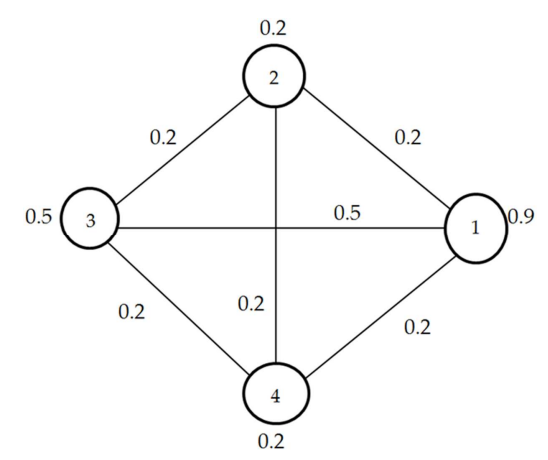

Figure 9. The fuzzy graph model $G$ for the traffic flow problem in Figure 8 based on Case 1.

Case 2: When $\mu(i, j) \ll \min \{\sigma(i), \sigma(j)\}$ for $i$ and $j$ with low traffic (where "«” represents much less than).

Suppose $\mu(2,4)=\varepsilon \ll 0.2$. So, the fuzzy graph model $G_{1}$ for the traffic flow problem in Figure 8 based on Case 2, is shown in Figure 10.

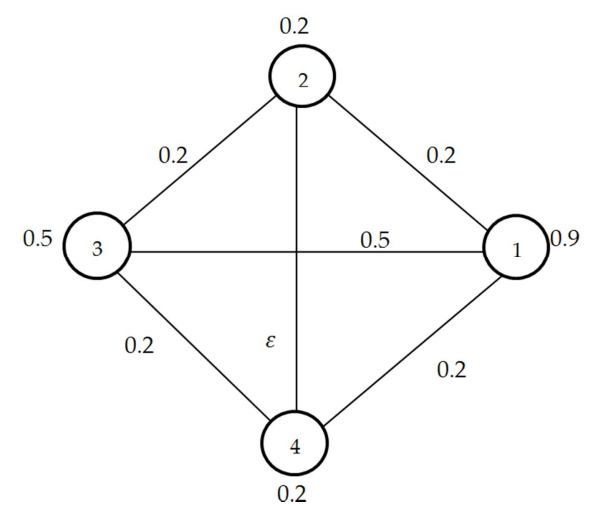

Figure 10. The fuzzy graph model $G_{1}$ for the traffic flow problem in Figure 8 based on Case 2.

Next, identify the types of arcs in the fuzzy graph models $G$ and $G_{1}$, since the fuzzy graph model $G$ shown in Figure 9 is a complete fuzzy graph, by Proposition 3.13 of [3] and Proposition 2 of [6], all arcs of a complete fuzzy graph are strong.

On the other hand, by routine computation, for the fuzzy graph model $G_{1}$ shown in Figure 10, we found that arc $(1,3)$ is $\alpha$-strong, arcs $(1,2),(1,4),(2,3)$ and $(3,4)$ are $\beta$-strong and $\operatorname{arc}(2,4)$ is $\delta$-arc. Hence, $\operatorname{arcs}(1,2),(1,3),(1,4),(2,3)$ and $(3,4)$ are the only strong $\operatorname{arcs}$ in $G_{1}$.

Now, determine the SFCP for the fuzzy graph model $G$ and $G_{1}$ based on their arcs. Since $G$ is a complete fuzzy graph, by Theorem $5, P_{s}^{f}(G, k)=P\left(K_{4}, k\right)$, where $K_{4}$ is the complete crisp graph.

But,

$$
P\left(K_{4}, k\right)=k(k-1)(k-2)(k-3)
$$

Therefore,

$$
P_{s}^{f}(G, k)=k(k-1)(k-2)(k-3)
$$

Analogously, since all the arcs in $G_{1}$ are not strong (b/c arc $(2,4)$ is $\delta$-arc). Suppose $H$ is a fuzzy subgraph obtained from the fuzzy graph $G_{1}$ by deleting $\delta$-arc. Explicitly, $H=G_{1}-$ $(2,4)$ is given in Figure 11 .

Therefore, by Theorem $2, P_{s}^{f}\left(G_{1}, k\right)=P\left(H^{*}, k\right)$ where $H^{*}$ is the underlying crisp graph of $H$ is shown in Figure 12.

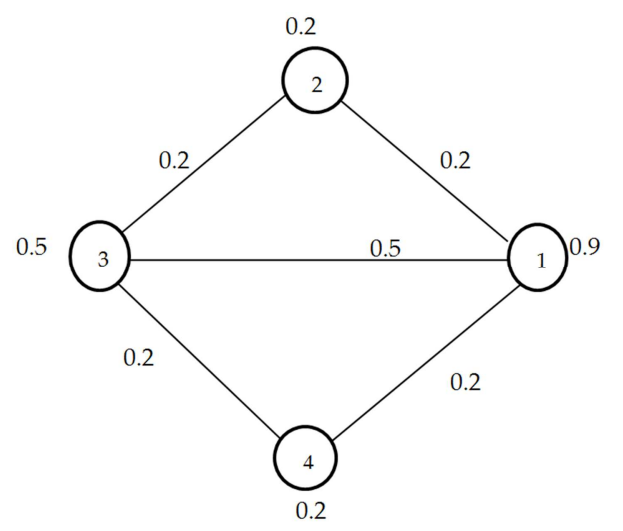

Figure 11. A fuzzy subgraph $H$ of $G_{1}$. 


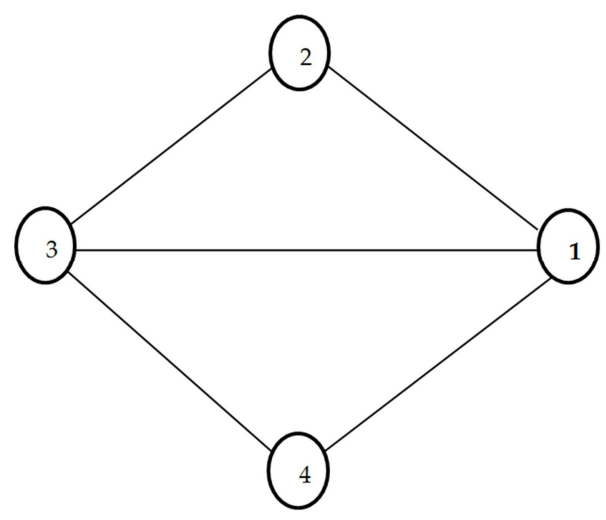

Figure 12. The underlying crisp graph $H^{*}$ of $H$.

By deletion contraction algorithm, we obtain,

$$
P\left(H^{*}, k\right)=k(k-1)(k-2)^{2}
$$

Hence,

$$
P_{s}^{f}\left(G_{1}, k\right)=k(k-1)(k-2)^{2}
$$

\section{Result and Discussion}

Consider the SFCP of $G$ in (14), and using Definition 23, we get, $\chi_{s}^{f}(G)=4$.

This result shows that there is a minimum of 4 traffic light phases are needed to control the traffic flow in Figure 8. With these 4 phases, the traffic flows at the intersection being smooth and safe.

Analogously, consider the SFCP of $G_{1}$ in (15), and using Definition 23,

we obtain, $\chi_{s}^{f}\left(G_{1}\right)=3$.

This result shows that there is a minimum of 3 traffic light phases are needed to control the traffic flow in Figure 8. For instance, in phase 1 , flow 1 can proceed, in phase 2 , flow 3 can proceed and in phase 3 , both flows 2 and 4 can go simultaneously at the intersection without making any conflict, however, the flows 2 and 4 have a conflicting movement originally.

In model $G$, the possibility of an accident occurs between each pair of conflict flows that are moving at the intersection is significant since all the flows are grouped into independent phases. But, in model $G_{1}$, the possibility of accident occurs between conflict flows 2 and 4 are insignificant (i.e. $\mu(2,4)=\varepsilon)$ since they are grouped in the same phase.

Therefore, the fuzzy graph model is very helpful and flexible for describing and handling the uncertain information in traffic flow problems and SFCP is a powerful mathematical tool for optimizing the traffic light phases at road intersections.

\subsection{Application for Obtaining the Possible Number of Traffic Light Patterns}

Most of the time, the traffic light pattern which we are going to plan at road intersection might not be appropriate, efficient and reduce the vehicle waiting time as compared with each other. Therefore, choosing an efficient and appropriate traffic light flow pattern with less waiting time is a major issue in traffic light control and management.
In this subsection, we apply SFCP to obtain the possible number of traffic light flow patterns with the optimized phases.

In Example 6, for the model $G$ in Figure 9, we have

$$
P_{s}^{f}(G, k)=k(k-1)(k-2)(k-3)
$$

And

$$
\chi_{s}^{f}(G)=4 .
$$

Therefore, $P_{s}^{f}\left(G, \chi_{s}^{f}(G)\right)=24$.

Here, the result shows that there are 24 possible traffic light flow patterns with 4 phases.

Similarly, for the model $G_{1}$ in Figure 10 , we have

$$
P_{s}^{f}\left(G_{1}, k\right)=k(k-1)(k-2)^{2}
$$

And

$$
\chi_{s}^{f}\left(G_{1}\right)=3
$$

Therefore, we obtain

$$
P_{s}^{f}\left(G_{1}, \chi_{s}^{f}\left(G_{1}\right)\right)=6
$$

Here, the result shows that there are 6 possible traffic light flow patterns with 3 phases.

Note that all of these traffic light flow patterns are nonconflicting combinations. The six possible traffic light flow patterns with 3 phases are depicted in Tables 1-6.

Table 1. Traffic Light Flow Pattern 1.

\begin{tabular}{ll}
\hline Phases & Proceed Flows \\
\hline Phase 1 & Only 1 \\
Phase 2 & Only 3 \\
Phase 3 & Only 2 \& 4 \\
\hline
\end{tabular}

Table 2. Traffic Light Flow Pattern 2.

\begin{tabular}{ll}
\hline Phases & Proceed Flows \\
\hline Phase 1 & Only 3 \\
Phase 2 & Only $2 \& 4$ \\
Phase 3 & Only 1 \\
\hline
\end{tabular}

Table 3. Traffic Light Flow Pattern 3.

\begin{tabular}{ll}
\hline Phases & Proceed Flows \\
\hline Phase 1 & Only 1 \\
Phase 2 & Only 2 \& 4 \\
Phase 3 & Only 3 \\
\hline & Table 4. Traffic Light Flow Pattern 4. \\
& Proceed Flows \\
\hline Phases & Only 3 \\
\hline Phase 1 & Only 1 \\
Phase 2 & Only 2 \& 4 \\
Phase 3
\end{tabular}

Table 5. Traffic Light Flow Pattern 5.

\begin{tabular}{ll}
\hline Phases & Proceed Flows \\
\hline Phase 1 & Only $2 \& 4$ \\
Phase 2 & Only 1 \\
Phase 3 & Only 3 \\
\hline
\end{tabular}


Table 6. Traffic Light Flow Pattern 6.

\begin{tabular}{ll}
\hline Phases & Proceed Flows \\
\hline Phase 1 & Only $2 \& 4$ \\
Phase 2 & Only 3 \\
Phase 3 & Only 1 \\
\hline
\end{tabular}

Computing the SFCP of the fuzzy graph at the minimum number of phases gives the possible number of traffic light patterns for the given traffic flow problem. Sometimes all the possible patterns which we have obtained are not equally appropriate and efficient. If this is the case, the SFCP is very helpful to choose the most appropriate and efficient patterns with reduced vehicle waiting times.

\section{Conclusion}

In this paper, we have introduced the concept of the strong fuzzy chromatic polynomial of fuzzy graphs and we have defined SFCP of fuzzy graph based on the strong coloring of a fuzzy graph. We have established the necessary and sufficient condition for SFCP of the fuzzy graph to be the chromatic polynomial of its underlying crisp graph. Furthermore, we have studied the SFCP of strong fuzzy graphs, complete fuzzy graphs, fuzzy cycles, and fuzzy trees. Also, we have given relations between SFCP and fuzzy chromatic polynomial of strong fuzzy graphs, complete fuzzy graphs, and fuzzy cycles. Finally, applications of SFCP theory to traffic flow problems are offered to demonstrate the applicability of the proposed work. In the future, we will develop a SFCP algorithm to solve strong coloring problems.

\section{References}

[1] Zadeh, L. A, Fuzzy sets. Inf. Control, vol. 8, No. 3, 1965, pp. 338-353.

[2] Rosenfeld, A, Fuzzy graphs, Fuzzy Sets, and Their Applications; Zadeh, L. A, Fu, K. S., Shimura, M., Eds.; Academic Press: New York, NY, USA, 1975; pp. 77-95.

[3] Bhutani, K, R., On automorphisms of fuzzy Graphs. Pattern Recognit. Lett. vol. 9, No. 3, 1989, pp. 159-162.

[4] Mordeson, J. N., and Nair, P. S. Cycles and cocycles of fuzzy graphs. Inf. Sci. vol. 90, 1996, pp. 39-49.

[5] Sunitha, M. S., and Vijayakumar, A. A., Characterization of fuzzy trees. Inf. Sci. vol. 113, 1999, pp. 293-300.

[6] Bhutani, K. R. and Rosenfeld, A., Strong arcs in fuzzy graphs. Inf. Sci. vol. 152, 2003, pp. 319- 322.

[7] Mathew, S. and Sunitha, M. S. Types of arcsin a fuzzy graph. Inf. Sci. vol. 179, No. 11, 2009, pp. 1760-1768.

[8] Mathew, S. and Sunitha, M. S. Strongest strong cycles and theta fuzzy graphs. IEEE Trans. Fuzzy Syst. vol. 21, No. 6, 2013, pp. 1096-1104.

[9] Mordeson, J. N.; Mathew, S. Advanced topics in fuzzy graph theory. Springer Nature: Gewerbestrasse 11, 6330 Cham, Switzerland, pp. 15-54, 2019.
[10] Talebi, A. A. Cayley fuzzy graphs on the fuzzy groups. Comput. Appl. Math. vol. 37, No. 4, 2018, pp. 4611-4632.

[11] Dhanyamol, M. V. On certain transit functions in fuzzy graphs Int. J. Uncertainty Fuzziness Knowl. -Based Syst. Vol. 25, No. 6, 2017, pp. 917-928.

[12] Tom, M. and Sunitha, M. S. Strong sum distance in fuzzy graphs. SpringerPlus vol. 4, No. 214, 2015, pp. 1-14.

[13] Mathew, S., Yang, H. L., and Mathew, J. K. Saturation in fuzzy graphs. New Math. Nat. Comput. Vol. 14, No. 1, 2018, pp. 113-128.

[14] Harinath, P. and Lavanya, S., Fuzzy graph Structures. Int. J. Appl. Eng. Res. vol. 10, 2015, pp. 70-74.

[15] Sitara, M., Akram, M., and Bhatti, M. Y., Fuzzy graph structures with application, Mathematics, vol. 7, No. 1, 2019, pp. 63.

[16] Akram, M. and Sitara, M., Certain fuzzy graph structures, J. Appl. Math. Comput., vol. 61, 2019, pp. 25-56.

[17] Akram, M., Bipolar fuzzy graphs, Inf. Sci. vol. 181, No. 24, pp. 5548-5564, 2011.

[18] Akram, M. m-polar fuzzy graphs: Theory, Methods \& Applications. Springer Nature: Gewerbestrasse 11, 633, Cham, Switzerland, pp. 7-112, 2019

[19] Verma, R., Merigo, J. M. and Sahni, M., Pythagorean fuzzy graphs: some results, arXiv: $1806.06721 \mathrm{v} 1$.

[20] Ashraf, S., Naz, S., and Kerre, E. E., Dombi fuzzy graphs. Fuzzy Inf. Eng. vol. 10, 2018, pp. 58-79.

[21] Akram, M., Dar, J. M., and Naz, S., Pythagorean Dombi fuzzy graphs. Complex Intell. Syst. 2019, pp. 1-26.

[22] Zuo, C., Pal, A., and Dey, A., New concepts of picture fuzzy graphs with application. Mathematics, vol. 7, No. 5, 2019, pp. 470 ,

[23] Akram, M. and Waseem, N., Novel applications of bipolar fuzzy graphs to decision-making problems. J. Appl. Math. Comput. vol. 56, 2018, pp. 73-91.

[24] Naz, S., Ashraf, S., and Akram, M., A novel approach to decision making with Pythagorean fuzzy information, Mathematics, vol. 6, No. 6, 2018, pp. 95.

[25] Akram, M. and Habib, A., Specific types of Pythagorean fuzzy graphs and application to decision making. Math. Comput. Appl., vol. 23, No. 3, 2018, pp. 42.

[26] Mordeson, J. N.; Mathew, S.; Malik, D. S. Fuzzy graph theory with applications to human trafficking. Springer International Publishing: Gewerbestrasse 11, 6330 Cham, Switzerland, pp. 181-107, 2018.

[27] Binu, M., Mathew, S. and Mordeson, J. N., Connectivity index of a fuzzy graph and its application to human trafficking. Fuzzy sets Syst. vol. 360, 2019, pp. 117-136.

[28] Binu, M., Mathew, S., and Mordeson, J. N., Wiener index of a fuzzy graph and application to illegal immigration networks. Fuzzy Sets Syst. In Press. 2019.

[29] Muñoz, S., Ortuño, M. T., Ramírez, J. and Yáñez, J., Coloring fuzzy graphs. Omega, vol. 33, No. 3, 2005, pp. 211-221. 
[30] Eslahchi, C. and Onagh, B. N., Vertex strength of fuzzy graphs. Int. J. Math. Math. Sci. vol. 2006 (Article ID 43614): 9, 2006.

[31] Kishore, A. and Sunitha, M. S., Chromatic number of fuzzy graphs. Ann. Fuzzy Math. Inform. vol. 7, No. 4, 2014, pp. 543 551 ,

[32] Samanta, S., Pramanik, T. and Pal, M., Fuzzy coloring of fuzzy graphs Afr. Mat. vol. 27, 2016, 2016, pp. 37-50.

[33] Mahapatra, T. and Pal, M., Fuzzy colouring of m-polar fuzzy graph and its application. J. Intell. Fuzzy Syst. vol. 35, 2018, pp. 6379-6391.

[34] Kishore, A. and Sunitha, M. S., Strong chromatic number of fuzzy graphs, Ann. Pure Appl. Math. vol. 7, 2014, pp. 52-60.

[35] Rosyida, I., Widodo, Indrani, Ch. R., Indriati, D., and Nurhaida. Fuzzy chromatic number of union of fuzzy graphs:
An algorithm, properties, and its application Fuzzy sets Syst. in Press, 2019.

[36] Mamo, A. A. and Srinivasa Rao, R. V. N., Fuzzy chromatic polynomial of fuzzy graphs with crisp and fuzzy vertices using $\alpha$-cuts. Advances in Fuzzy Systems, vol. 2019, Article ID 5213020, 11 pages.

[37] Mordeson, J. N. and Nair, P. S., Fuzzy Graphs and Fuzzy Hypergraphs; Springer: Heidelberg, Germany, pp. 19-39, 2000.

[38] Mathew, S., Mordeson, J. N. and Malik, D. S., Fuzzy graph Theory, Springer International Publishing: Gewerbestrasse 11, 6330 Cham, Switzerland, pp. 13-57, 2018.

[39] Nagoorgani, A., Isomorphism properties on strong fuzzy graphs. Int. J. Algorithm Comput. Math. vol. 2, No. 1, pp. 3947, 2009. 